\title{
Review Paper on Navigation System for Visually Impaired People
}

\author{
Chaitali K. Lakde ${ }^{1}$, Dr. Prakash S. Prasad ${ }^{2}$ \\ P.G. Student, Department of Computer Technology, Priyadarshini College of Engineering, Nagpur, India ${ }^{1}$
}

Associate Professor and Head of Dept., of Computer Technology, Priyadarshini College of Engineering, Nagpur, India ${ }^{2}$

\begin{abstract}
Mobility of visually impaired people is restricted by their incapability to recognize their surroundings. According to the World Health Organization (WHO) in 2012, out of 7 billion global population there were over 285 million visually impaired people and 39 million were totally blind out of which 19 million are children ( below 15 years). This means that someone in our world goes blind in every five seconds and a child in every minute. Over 90 percent blind children obtain no schooling. Recent survey source India is now became the world's large number of blind people. The population of India has reached $120 \mathrm{Cr}$. of those $8.90 \mathrm{Cr}$. people are visually impaired. $90 \%$ of those cannot travel independently. In this paper, we present a survey of navigation system of visually impaired people highlighting various technologies with their practical usefulness, design and working challenges and requirements of blind people. The aim of this paper is to provide a better understanding to identify important research directions in this increasingly important social area for future research.
\end{abstract}

Keywords: Navigation system, visually impaired, obstacle detection, mobility, localization, indoor navigation, outdoor navigation, GPS, RFID, ultrasonic sensor

\section{INTRODUCTION}

Visually challenged persons face constraints in independent mobility and navigation. Mobility means the possibility of liberally moving, without support of any supplementary person, at home and unfamiliar scenarios. People with visual impairment tackle enormous limitations in terms of mobility.

A system which guide or assist people with vision loss, ranging from partially sight to totally blind, by means of sound commands is referred as Navigation assistance for visually impaired (NAVI). Many researches are being conducted to build navigation system for blind people. Most of these technologies have limitations as its challenge involves accuracy, usability, interoperability, coverage which is not easy to overcome with current technology for both indoor and outdoor navigation.

\section{RELATED WORK}

\section{A. Problems Faced by Visually Impaired People}

Traditionally white cane is the most popular, simplest tool for detecting obstacles due to its low cost, portability. It enables user to effectively scan the area in front and detect obstacles on the ground like holes, steps, walls, uneven surfaces, downstairs etc .but it can only be used to detect obstacles up to knee-level. Its detection range is limited up to 1-2 feet only. Certain obstacles (e.g. protruding window panes, raised platforms, a moving vehicle, horizontal bars) cannot be detected till they are dangerously close to the person. Even dog guides are very capable to guide these persons but they are unable to detect potentially hazardous obstacles at head level. Guide dog service stage is on average 6 years and requires regular dog up-keeping expenditure and lifestyle changes. [1]

Several solutions have been proposed in the recent years to increase the mobility and safety of visually impaired persons.

A system "Roshni" determines the user's position in the building, navigation via audio messages by pressing keys on the mobile unit. It uses sonar technology to identify the position of user by mounting ultrasonic modules on ceiling at regular intervals. This system is portable, easy to operate and is not affected by environmental changes. But this system is limited only for indoor navigation because it requires detailed interior map of the building. [2]

RFID based map-reading system which provides technical solution for the visually impaired to pass through public locations easily using RFID tag grid, RFID cane Reader, Bluetooth interface and personal digital assistance. But its initial development cost is quite high and chances of interference in heavy traffic. [3]

A voice operated outdoor navigation system developed using GPS, voice and ultrasonic sensor. It can alert user's current position and provide verbal guidelines for travelling to a remote destination but fails to give obstacle detection and warning alert. [4]

Another real-time technology developed to alert visually impaired user by the presence of static / dynamic obstacles in a few meters surrounding, which works without depending on any Smartphone, uses camera for background motion detection. This system is robust to complex camera and background motion and does not required any prior knowledge about the obstacle size, 
shape or position. This camera based image processing system can be a better option but it requires lot processing power and hence system becomes bulky, costly and it must be transportable. [5]

\section{Use Scenario Indoor/Outdoor}

Navigation system generally comprised either an indoor or outdoor positioning system or both, for detecting the position of consumer. Most of the outdoor navigation systems employ GPS for positioning. Unfortunately, GPS can only be used outside of buildings because the employed radio signals cannot penetrate solid walls.

Outdoor navigation systems generally rely upon GPS; indoor systems rely upon different techniques for localizing the consumer, as GPS signals cannot be received indoors. Currently, indoor navigation systems always employ radio signal for positioning, which may suffer from the problem of signal impairments, such as multipath propagation and Radio Frequency interference.

A navigation system for blind pedestrian using RFID passive tags provides location markers. A mobile receiver provides navigation guidelines based on the code saved in each tag. This system overcomes the GPS navigation limits in indoor environment and does not need any power supply for location markers. On the counter side, the tags assignment cost could be high and generally tags are not easy to adjust for an outdoor, insensitive environment. [6]

Most of the recent years research for outdoor navigation uses Global Positioning System (GPS) based on geostationary satellite signals. These tools may integrate other services such as Geographic Information Systems (GIS). An extraordinary blind navigation system proposed based on GIS, GPS and wireless technology. But the resolution of GPS localization is limited for civil purposes (few meters). Again this system has issue of delay in the response when the receivers are used. Because of this low real-time responsiveness and lack of accuracy, system lacks behind to provide needed level of safety for visually impaired person. [7]

Another system for visually impaired person gives a structure using IR sensor and magnetic compass on handheld device. This system determines location and orientation of the user though a voice enabled GPS inside a closed surrounding. .But this system is limited for indoor navigation. [8]

\section{Some hybrid systems used in indoor as well as outdoor} mode

A talking assistance type location finding system proposed for both indoor and outdoor navigation. System consists of walking stick having GSM module to send message to authorized person at the time of tragedy, sonar sensors and RF transmitter and receiver. For indoor localization RFID and for outdoor localization GPS system is used. Thus, this GPS system used in walking cane reduces the cost of installing many RFID tags in outdoor to identify the place. [9]

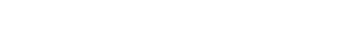

GPS based technique is "Drishti" which can switch the system from an indoor to an outdoor environment and vice versa with a simple vocal command. To provide complete navigation system, authors extend indoor version of Drishti to the outdoor versions for blind pedestrians by adding only two ultrasonic transceivers that are smaller than a credit card and are tagged to the user's shoulder. System provides a real-time communication between user and the mobile client via the headphone in which user can ask for the path, obstacle prompts, and even his/her current location in familiar or unfamiliar surrounding also. Unfortunately, this system has two limitations. As only two beacons attached to the user's shoulder, so it becomes impossible to obtain the height data of the user. Used algorithm calculates the location of user in two dimensions assuming the average height of a person, which gives larger error if the user sits or lies down. Another limitation is that because of signals reflection or blocking by walls and furniture, there are some "dead spots" due to the bad faulty date reads. [10]

\section{E. Different gadgets available in different scenarios}

Wearable and portable assistive technologies are also used for supporting people with disabilities such as the blind. Wearable devices are allowing hands-free interaction, or at least minimizing the use of hands when using the device, while portable assistive devices required a constant hand interaction.

A wearable obstacle avoidance electronics device designed to serve the navigation system of visually impaired person. System consist of implementation of the vOIce-"seeing with sound" system which contains glasses with attached camera, portable computer and ear speakers. System emphasizes its characteristics like free hands, free ears, wearable and east to operate. [11]

An ultrasonic sensor based navigation system for blind people is based on microcontroller with synthetic speech output and portable device to guide the user about urban walking paths to point out what decisions to make. This device uses the principle of reflection of high frequency ultrasonic beam to detect obstacles in the path. This mobility support instructions are given by vibro-tactile form in order to reduce navigation difficulties. A disadvantage of ultrasound is that walls may reflect or block ultrasound signals, which result in less accurate localization. [12]

An indoor navigation system for visually impaired people constantly tracks the user through an RFID unit and communicated the user to obtain desired destination safely via wireless connection and through a tactile compass. [13]

"Blind audio Guidance system" is based on embedded system, uses ultrasonic sensor for distance measurement, IR sensor for object detection and AVR sound system for audio instructions. The main functions of this system are 
environment recognition and path detection. Ultrasonic sensors receive visual information and this visual information is transformed into auditory information. To represent the information about the position of obstacles audio components of intensity, frequency, binaural phase difference are used. This signal transformation system reduces the training time required to use a white cane. However, only issue of this system is the difficulty to know one's location globally. [14]

Vibration and voice operated navigation system developed using ultrasonic sensors to detect obstacles. Since visually impaired people are more sensitive in hearing and possesses strong perception than ordinary people. So this system gives alert through vibration and voice feedback. System works in indoor as well as outdoor navigation and focus on continuously sensing surround obstacles and alerting through vibration and voice feedback. Depending upon the distance between obstacle and user different intensity levels are provided to vibration motor to alert user's mobility.

A navigation system designed for blind people using RGB-D sensor with range expansion. System uses a consumer RGB-D camera for range and visual information, which support range based floor segmentation. Cheaper RGB sensor supports in object detection and color sensing. User interface is given through audio instructions and sound map information. [16]

\section{III.CONCLUSION}

This paper provides a broad overview of state of the art techniques used for navigation system for visually impaired people. It concludes that navigation system have not achieved large scale exploitation mainly due to unaffordable cost, accuracy, usability. Future navigation system needs to first and foremost lower the installation expenditure by minimizing the infrastructure disputes that is required for localizing the consumer. Usability needs to be improved by minimizing the amount of sensors users have to carry and providing usable directions in a robust modality of feedback. System need to take into account the user's special requirements, minimize cognitive load, cost effective, user friendliness and minimize any interference from the environment.

\section{REFERENCES}

[1] B. B. Blasch, W. R. Wiener, and R. L. Welsh, Foundations of Orientation and Mobility, 2nd ed. New York: AFB Press, 1997.

[2] Roshni: Indoor Navigation System for Visually Impaired by D.Jain and M.Balakrishnan, P.V.M.Rao.

[3] P.Dharani, B.Lipson and D.Thomas, "RFID Navigation System for the Visually Impaired", Worcester Polytechnic Institute, 2012.

[4] S.Koley and R.Mishra, "Voice operated outdoor navigation system for visually impaired persons", International Journal of Engineering Trends and Technologyvol. 3, issue2- 2012.

[5] R. Tapu, B. Mocanu, T. Zaharia " Real time static/dynamic obstacle detection for visually impaired persons" IEEE International Conference on consumer electronics (ICCE),978-1-4799-21919/14, pp. 394-395,2014.
[6] V. Kulyukin, C. Gharpure, J. Nicholson, S. Pavithran, "RFID in Robot- Assisted Indoor Navigation for the Visually Impaired", Proceedings of 2004 lEEE/RSJ International Conference on Intelligent Robots and Systems, September 28 -October 2,2004, Sendai, Japan.

[7] Z. Hunaiti, V, Garaj, W. Balachandran, F. Cecelja, "An Assessment of $3 \mathrm{G}$ link in a Navigation Systems for Visually Impaired Pedestrians", Proceedings of the 15th International Conference on Electronics, Communications and Computers, 2005,pp. 180-186.

[8] P.Shah, P.Mehta, P.Kant and A.K.Roy, "VI-Navi: A Novel Indoor Navigation System for Visually Impaired People".

[9] Nandhini.N, Vinoth chakkaravarthy.G , G.Deepa Priya, "Talking Assistance about Location Finding both Indoor and Outdoor for Blind People", International Journal of Innovative Research in Science, Engineering and Technology, volume 3, February 2014, pp. 9644-9651.

[10] Lisa Ran, Sumi Helal and Steve Moore, "Drishti: An Integrated Indoor/Outdoor Blind Navigation System and Service", Proceedings of the Second IEEE Annual Conference on Pervasive Computing and Communications 2004 IEEE.

[11] Dimitrios Dakopoulos and Nikolaos G. Bourbakis, "Wearable Obstacle Avoidance Electronic Travel Aids for Blind: A Survey", IEEE Transactions on Systems, man, and cybernetics- part c: application and reviews, volume 40, January 2010.

[12] Mounir Bousbia-Salah , Abdelghani Redjati, Mohamed Fezari, Maamar Bettayeb, "An Ultrasonic Navigation System For Blind People", IEEE International Conference on Signal Processing and Communications (ICSPC 2007),Dubai,24-27November2007,pp. 1003-1006.

[13] Rusen Oktem, Elif Aydın, Nergiz Ercil Cagıltay, "An Indoor Navigation Aid Designed for Visually Impaired People", IEEE, 2014, pp. 2982-2987.

[14] Arjun Sharma, Rahul Patidar, Shubham Mandovara, Ishwar Rathod, "Blind Audio Guidance System", International Journal of Emerging Technology and Advanced Engineering, volume 3, January 2013,pp.17-19.

[15] N.Mahmud, R.K.Saha, R.B. Zafar, M.B.H. Bhuian, and S.S.Sarwar, "Vibration and Voice Operated Navigation System for Visually Impaired Person", 3rd International Conference on Informatics, Electronics \& Vision, 2014.

[16] A. Aladren, G. Lopez-Nicolas, Luis Puig, and Josechu J. Guerrero, "Navigation Assistance for the Visually Impaired Using RGB-D Sensor With Range Expansion", 2014 IEEE.

\section{BIOGRAPHIES}

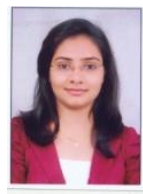

Chaitali K. Lakde received undergraduate degree in electronics engineering in 2013. She has presented a national conference.

She is currently student of M.E. in wireless communication and computing branch.

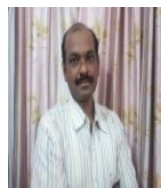

Dr. Prakash S. Prasad obtained Ph.D. degree in Computer Science \& Engineering. $\mathrm{He}$ has published more than 16 papers in National and International Conferences. He is Member of IEEE, ISTE and IACSIT. He has completed his bachelors degree in 1997, and Masters Degree in 2007.

$\mathrm{He}$ is currently working as Associate Professor at Priyadarshini college of Engineering and Head of The Department of Computer Technology. He is having 16 Years of Teaching experience and his interests include network security, Operating System and System Software. 